\title{
Castleman disease with rapidly progressive pemphigus: a case report
}

\author{
Ruiying $\mathrm{Fu}^{\mathrm{a}}$, Yingzhi $\mathrm{He}^{\mathrm{b}}$, Liang Wang ${ }^{\mathrm{a}, *}$ \\ ${ }^{a}$ Department of Hematology, Beijing Tongren Hospital, Capital Medical University, Beijing 100730, China. \\ ${ }^{b}$ Zhujiang Hospital of Southern Medical University, Guangzhou 510280, China.
}

\begin{abstract}
Paraneoplastic pemphigus (PNP) is a rare autoimmune disorder of the skin, which is associated with various underlying benign or malignant neoplasms. We report a case of a 34-year-old man with rapidly progressed and extensive skin lesions, and significant weight loss. He was diagnosed as pemphigus Vulgaris with a skin biopsy firstly, then the PET/CT scan showed a retroperitoneal mass. Pathological examination of the retroperitoneal mass confirmed the diagnosis of Castleman disease with paraneoplastic pemphigus. By reporting this case, we aimed to improve the clinical alertness and awareness of Castleman disease in patients characterized by paraneoplastic pemphigus.
\end{abstract}

Keywords: Paraneoplastic pemphigus, Castleman disease, paraneoplastic syndrome, hematologic malignancies

\section{Introduction}

Among many cases of autoimmune dermatological disorders, paraneoplastic pemphigus shows a difference due to their antigenic specificity. It was first described in 1990 by Anhalt, and polymorphous mucous lesions, especially in the oral cavity, were common in all cases. In most cases, PNP is associated with an underlying neoplasm which comes to light as of the skin lesions. Up to now, most of those PNP cases are caused by hematologic neoplasms or disorders, and lymphoproliferative disorders are the most frequent diseases associated with PNP, such as nonHodgkin's lymphoma, chronic lymphocytic leukemia, Castleman disease, and Waldenstrom's macroglobulinemia. Moreover, carcinomas from epithelial cells and sarcomas from mesenchymal cells also have been reported in some cases of PNP. The prognosis of PNP is usually poor, systemic complications, including sepsis, gastrointestinal bleeding, and bronchiolitis obliterans, account for the majority of deaths. Thus, the effective control of the oral and skin lesions, an adequate treatment of the underlying neoplasm, and prevention of bronchiolitis obliterans could be

\footnotetext{
* Corresponding author: Liang Wang
}

Mailing address: Department of Hematology, Beijing Tongren Hospital, Capital Medical University, Beijing, 100730, China. Email: wangliangtrhos@126.com

Received: 16 November 2020 / Accepted: 11 December 2020 extremely beneficial to the patient's prognosis. Here, we presented a unique case of PNP associated with Castleman disease, a 34-year-old man who came to us with refractory polymorphous skin lesions. Through a combination of skin biopsy and retroperitoneal mass biopsy, this patient was finally diagnosed with Castleman disease with PNP. Informed consent for publication of this case was obtained from the patient.

\section{Case report}

A 34-year-old man presented to our outpatient clinic with a 2-week history of rapid-onset skin lesions affecting the whole body and skeletonization appearance. As is shown in Figure 1A, physical examination revealed multiple ulcer lesions with blood crust involving the oral cavity, lips, eyelid, conjunctiva, trunk, genitalia, limbs, hands, and feet. Some blisters with turbid fluid in them can be found around fingers and shoulders. Figure 1B showed the appearance of this patient about 2 months before disease onset, with about 100 kilograms of bodyweight, which decreased to 60 kilograms now. A skin biopsy confirmed the diagnosis of pemphigus Vulgaris with intraepidermal blisters and depositions of IgG. Serologic testing showed antibodies against desmoglein 3 , while other autoantibodies including antinuclear antibodies were negative. Bone marrow aspiration and biopsy examination did not reveal positive findings. A PET-CT scan was performed, which revealed a retroperitoneal mass $(4 \mathrm{~cm} \times 5 \mathrm{~cm}$ size $)$ closely around the abdominal aorta with a standard uptake value 


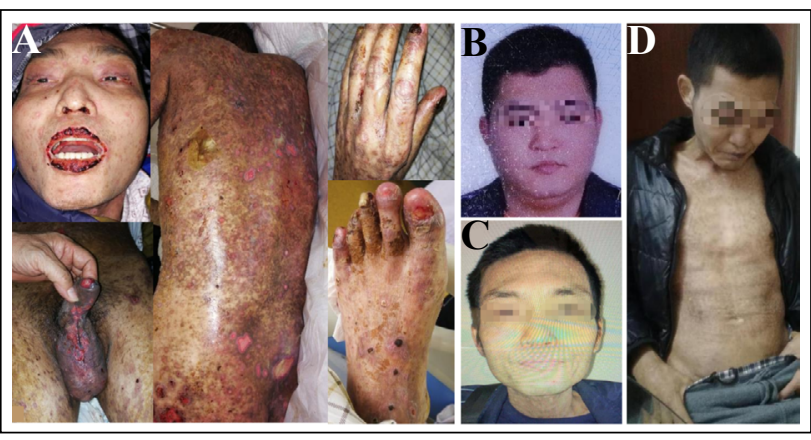

Figure 1. (A) Physical examination revealed multiple ulcer lesions with blood crust involving the oral cavity, lips, eyelid, conjunctiva, trunk, genitalia, limbs, hands, and feet. Some blisters with turbid fluid in them can be found around fingers and shoulders. (B) The appearance of this patient about 2 months before disease onset, with about 100 kilograms of body weight. (C) The appearance after about 1-year exposure to lowdose prednisone, with all skin lesions healed but still skinny. (D) The disease remained stable thereafter.

of 7. The laparoscopic mass biopsy was done twice (the first one failed to get any meaningful tissues), and the pathologic examination confirmed the diagnosis of hyaline vascular type of unicentric Castleman disease. Thus, this patient was finally diagnosed with Castleman disease with paraneoplastic pemphigus. Due to the extremely high risk of complete surgical excision, palliative treatment with prednisone was given. Figure $1 \mathrm{C}$ showed the appearance after about 1-year exposure to low-dose prednisone, with all skin lesions healed but still skinny. Then prednisone was stopped, and as is shown in Figure 1D, no obvious change occurred thereafter. A CT scan was done and the retroperitoneal mass still existed with no significant enlargement. Castleman disease is the most common underlying disease that causes paraneoplastic pemphigus (PNP), and complete surgical resection of a tumor mass can successfully control PNP.

\section{Discussion}

Paraneoplastic pemphigus (PNP) is a rare autoimmune skin disease belonging to the group of blistering diseases, which is always associated with a concealed neoplasm. Generally, oral, skin and mucosal lesions are the earliest manifestations shown by patients with PNP. PNP is always characterized by an association with neoplasms, including carcinoma of the stomach, lung, and colon $[1,2]$. Besides, B-cell hematological malignancies are most frequently reported in association with PNP [3, 4]. Castleman disease is a heterogeneous nonmalignant lymphoproliferative disorder. Major subtypes of Castleman disease include unicentric versus multicentric presentation; hyaline vascular, plasmacytic, or mixed pathology; and HHV8-associated (typically HIV-positive) versus idiopathic disease [5]. What we know is that both auto-antibodies and cell-mediated immunity play an important role in the pathogenesis of PNP [3, 4, 6, 7]. The most common autoantibodies detected in PNP were directed against the plakin family and PNP antibodies mostly belong to the
IgG class. Similarly, the detection of immunocytes like CD8+ T-cells and MHC-restricted CD8+ cytotoxic T cells indicates the activation of the cell-mediated immune system. Castleman disease is involved in the production of autoantibodies in patients with PNP, which is important in the pathogenesis of the disease. Researches indicate that the expansion of several immunologically active B-cell clones arises in Castleman disease, and these clones can secrete autoantibodies that react specifically with epidermal proteins, which lead to impairment of cell-cell adhesion and subsequent development of pemphigus lesions in the skin and mucosal membranes [8]. Surgical resection of the tumor is necessary for patients with Castleman disease and PNP, and the complete removal of the tumor may induce remission or full regression of the disease, with the decrease in antibody titer [9]. High doses of corticosteroids, radiation, chemotherapy, and immunosuppressive therapy have been used as alternative treatments. In our case, in consideration of the high risk of surgery, palliative treatment with prednisone was given. The retroperitoneal tumor mass shrank obviously at first but remained stable thereafter. Although all skin lesions gradually healed, the patient still seems skinny after about one year of treatment with low-dose prednisone.

\section{Declarations}

Authors' contributions: RYF and YZH collected the data and treated the patient. LW analyzed the data and wrote the paper. All authors have read and approved the final version of the paper.

Conflict of interest: The author declares no conflict of interest.

Consent for publication: Informed consent for publication of this case was obtained from the patient.

\section{References}

1. Paolino G, Didona D, Magliulo G, et al. Paraneoplastic pemphigus: insight into the autoimmune pathogenesis, clinical features and therapy. International Journal of Molecular Sciences, 2017, 18(12): 2532.

2. Kelly S, Schifter M, Fulcher D A, et al. Paraneoplastic pemphigus: Two cases of intra-abdominal malignancy presenting solely as treatment refractory oral ulceration. The Journal of Dermatology, 2015, 42(3): 300-304.

3. Sehgal V N, Srivastava G. Paraneoplastic pemphigus/ paraneoplastic autoimmune multiorgan syndrome. International journal of dermatology, 2009, 48(2): 162169.

4. Baum S, Sakka N, Artsi O, et al. Diagnosis and classification of autoimmune blistering diseases. Autoimmunity reviews, 2014, 13(4-5): 482-489.

5. Abramson J S. Diagnosis and Management of Castleman Disease. Journal of the National Comprehensive Cancer 
Network, 2019, 17(11.5): 1417-1419.

6. Anhalt G J, Kim S C, Stanley J R, et al. Paraneoplastic pemphigus: an autoimmune mucocutaneous disease associated with neoplasia. New England Journal of Medicine, 1990, 323(25): 1729-1735.

7. Nguyen V T, Ndoye A, Bassler K D, et al. Classification, clinical manifestations, and immunopathological mechanisms of the epithelial variant of paraneoplastic autoimmune multiorgan syndrome: a reappraisal of para- neoplastic pemphigus. Archives of Dermatology, 2001, 137(2): 193-206.

8. Wang L, Bu D, Yang Y, et al. Castleman's tumours and production of autoantibody in paraneoplastic pemphigus. The Lancet, 2004, 363(9408): 525-531.

9. Lemon M A, Weston W L, Huff J C. Childhood paraneoplastic pemphigus associated withCastleman's tumour. British Journal of Dermatology, 1997, 136(1): 115-117.

Cite this article as: Fu R, He Y, Wang L. Castleman disease with rapidly progressive pemphigus: case report[J]. Aging Pathobiology and Therapeutics, 2020, 2(4): 223-225. 